Unfallchirurg 2010 $1113: 257$

DOI 10.1007/s00113-010-1761-4

Online publiziert: 20. März 2010

(c) Springer-Verlag 2010

F. Gebhard ${ }^{1}$. U. Culemann ${ }^{2}$

${ }^{1}$ Klinik für Unfallchirurgie, Hand-, Plastische und Wiederherstellungschirurgie, Zentrum für Chirurgie, Universitätsklinikum Ulm

${ }^{2}$ Chirurgische Klinik, Universitätsklinikum des Saarlandes, Homburg/Saar

\title{
Beckenringverletzungen im Alter
}

Liebe Kolleginnen und Kollegen,

Beckenverletzungen haben grundsätzlich vom Schweregrad her ein weites Spektrum. Dieses reicht von einfachen, nichtdislozierten Frakturen mit einem rein osteoligamentären Problem bis hin zu lebensbedrohlichen Komplexverletzungen mit begleitender Weichteilsymptomatik und entsprechend aufwendigem Management.

Die sich in Deutschland verschiebende Alterspyramide bedingt auch eine Zunahme von Beckenverletzungen mit höherem Instabilitätsgrad (B/C-Verletzungen) im Alter. Während früher fast ausschließlich junge männliche Patienten ein komplexes Beckentrauma infolge eines Hochrasanztraumas erlitten, kommt dieses Verletzungsmuster zunehmend auch bei älteren, weiblichen Patienten infolge eines banalen Sturzes auf die Seite (Niedrigenergietrauma) vor.

\section{( Klassische}

Versorgungskonzepte

bei Beckenfrakturen

basieren auf einer guten

\section{Knochenqualität}

Klassische Versorgungskonzepte bei Beckenfrakturen basieren auf einer guten Knochenqualität, die eine standardisierte, osteosynthetische Versorgung ermöglicht. Osteoporose, Tumorleiden und andere Begleiterkrankungen mit Dauermedikamentenpflicht führen dazu, dass die technischen und chirurgischen Anforderungen an die Frakturversorgung älterer Patienten deutlich höher sind als früher. Dies drückt sich auch in zahlreichen Modifikationen der Standardversorgungen aus.

Neben der zumeist deutlich erschwerten Anamnese und Diagnostik ergeben sich bei zunächst stabiler Fraktursituation im Verlauf immer wieder auch sekundär auftretende Dislokationen, die bei Nichtbeachtung in schmerzhafte Pseudarthrosen mit dauerhafter Immobilität des Patienten münden können. Diesen Verläufen muss die Behandlung des alten Patienten während des gesamten Verlaufs Rechnung tragen und durch entsprechende Überprüfungen (klinische Verlaufskontrollen, engmaschigere CT-Kontrolle) vermieden werden. Starke Schmerzen sollten hierbei ein entsprechendes Warnsignal sein!

Die folgenden Beiträge sollen diese Thematik durch Darstellung der Management- und Versorgungskonzepte im Alter näher beleuchten. Selbstverständlich können damit nicht alle Aspekte vollständig abgebildet werden, weshalb auf die inzwischen immer breiter aufgestellte Literatur zu diesem komplexen Thema bereits an dieser Stelle ausdrücklich hingewiesen wird.

Der Arbeitsgruppe Becken der Deutschen Gesellschaft für Unfallchirurgie sei an dieser Stelle Dank ausgesprochen für die jahrelange, erfolgreiche Dokumentation und Auswertung dieses Frakturbildes.

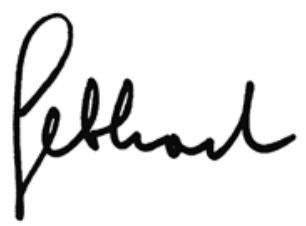

Prof. Dr. F. Gebhard, Ulm

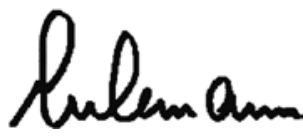

PD Dr. U. Culemann, Homburg/Saar

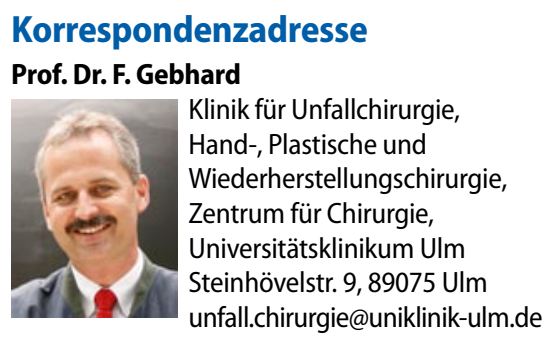

